THE ROLE OF LIVER TRANSPLANTATION IN SYSTEMIC AMYLOIDOSIS

L. B.Lovar ${ }^{1}$ M. R. Persey ${ }^{1}$, A. J. Stangou ${ }^{2}$, M. Rela ${ }^{2}$, N. Heaton ${ }^{2}$, R. Williams ${ }^{2}$, S. Madhoo', J. A. C. Buckels ${ }^{3}$, M. B. Pepys ${ }^{1}$, P. N. Hawkins'. ' ${ }^{1}$ Royal Postgraduate Medical School and ${ }^{2}$ Institute of Liver Studies, King's College Hospital, London and Liver Unit, ${ }^{3}$ Queen Elizabeth Hospital, Birmingham

The systemic amyloidoses are a group of progressive and usually fatal multi-organ diseases. We have developed ${ }^{123}$ I-serum amyloid $P$ component ( ${ }^{123}$ I-SAP) as a specific nuclear medicine tracer with which the amyloid deposits can be imaged quantitatively in vivo. Scans have shown hepatic amyloid in $\mathbf{4 8 \%}$ of 200 patients with $\mathrm{AL}$ amyloidosis and $18 \%$ of 92 patients with AA amyloidosis. Liver amyloid, however, was not a feature in 47 patients with familial amyloid polyneuropathy (FAP) associated with variant transthyretin (TTR) even though the liver is the only source of the genetically variant amyloid precursor protein in the plasma.

Massive hepatic amyloidosis was common in our series but clinically significant liver failure was very rare. We report here the first cases to undergo orthotopic liver transplantation (OLT) for liver failure due to amyloid, and the outcome of OLT in patients with FAP associated with variant TTR in whom surgery was performed to correct the inherited metabolic error. OLT was successful in a 44 year old man with progressive liver failure due to $\mathrm{AL}$ and in a 15 year old boy with hereditary lysozyme amyloidosis that caused acute hepatic rupture. They are alive after 4 and 18 months respectively with normal hepatic function and with no evidence as yet of recurrent liver amyloid.

Following OLT in FAP, prospective serial SAP scans over 1-3 years have shown regression of renal and splenic amyloid deposits in 7 out of 8 cases. This has been associated with stabilisation of peripheral neuropathy and substantial improvement in autonomic and gastrointestinal function in most cases.

OLT is not only potentially curative in FAP, but may also improve the survival of selected patients with amyloid related liver failure.

\section{POSTTRANSPLANT CHRONIC HEPATITIS IN FULMINANT HEPATIC FAILURE}

RMohamed, DF Mirza, BK Gunson, DJ Mutimer, SG Hubscher. Liver Unit, Queen Elizabeth Hospital, University of Birmingham,UK.

Non-A non-B (NANB) or seronegative hepatitis is the leading indication for liver transplantation in patients with fulminant hepatic failure (FHF). We attempted to define the extent of histological changes in protocol annual liver allograft biopsies between 1989 and 1994 in adult patients transplanted for FHF.

111 biopsy specimens from 41 patients with fulminant NANB hepatitis and 34 from a control group of 16 patients with other causes of FHF (11 paracetamol overdose, 2 idiosyncratic drug reaction, 3 Wilson's disease) were available. Specimens were analysed using standard proforma without knowledge of the original diagnosis. The median follow-up of 36 months was the same in the two groups of patients.

Chronic hepatitis was present in at least 1 biopsy in 29/41 (71\%) patients with fulminant NANB hepatitis compared to $5(31 \%)$ patients in the control group. Inflammatory grade was mild in 22, moderate in 3 and severe in 4 patients with NANB FHF in contrast to mild hepatitis in all 5 patients who had other causes of FHF. 21 (51\%) patients with fulminant NANB hepatitis had fibrosis, 10 mild (periportal fibrosis without bridging, 8 moderate (bridging fibrosis without nodule formation) and 3 severe (cirrhosis) compared to 4 (25\%) mild fibrosis in the control group. The median aspartate transaminase (normal 5-30u/L) at the time of biopsy was $24(11-316 u / L)$ in NANB vs $17(15-35 u / L)$ in the latter, $p=n s$.

Excluding early allograft failure, 6 patients with NANB FHF required retransplantation ( $2=$ chronic rejection, $1=$ severe hepatitis with panacinar necrosis, resembling original liver, $3=$ chronic hepatitis with pre-cirrhotic fibrosis and prominent cholestasis of unknown aetiology). One patient in the control group had a second graft (chronic rejection).

Conclusion: The occurrence and severity of chronic hepatitis is high following transplantation for seronegative hepatitis. Graft survival may be adversely influenced by the development of chronic hepatitis, which may represent persistent or recurrent disease.
PERIPHERAL EOSINOPHILIC COUNT BOTH BEFORE AND AFTER LIVER TRANSPLANTATION REFLECTS THE PRESENCE OR ABSENCE OF ACUTE REJECTION. MM Dollinger, JN Plevris, OJ Garden, PC Hayes. Scottish Liver Transplant Unit, The Royal Infirmary, Edinburgh, EH3 9YW, Scotland, UK.

Introduction: Acute cellular rejection (ACR) is common following orthotopic liver transplantation (OLT) and is an important cause of graft dysfunction. Eosinophils, potent mediators of tissue damage, have been implicated in the pathogenesis of ACR. Aims and methods: We studied 55 OLT patients all of whom had a protocol biopsy 7 days post-OLT and whose peripheral eosinophilic count was monitored daily for 11 days following OLT. Patients were divided clinicopathologically into two groups; group (A) without rejection, group (B) with rejection. Results: $36 \%$ of patients (group B) developed ACR within the 11 day study period. The pre-transplant EC was significantly higher in group B, comparing with group A $(0.31 \pm 0.08$ vs $0.10 \pm 0.01$ (x 10 $1 /)$, $p<0.001)$. Following OLT, EC fell to low levels in both groups. By day 3 there was a statistically significant rise in the EC in group B compared with group $A$, which was maximum at day $7[0.51 \pm 0.06$ vs $0.26 \pm 0.03$ $\left.\left(x 10^{9} / 1\right) p<0.001\right]$. Following treatment with steroids, EC dropped to similar values as in group $A$ and remained low thereafter in 16/20 patients. Four patients had a 2nd episode of ACR; in all four, EC was raised again and decreased with resolution of the rejection. An EC threshold of $0.13\left(\times 10^{9} /\right)$ before OLT predicted subsequent development of ACR (sensitivity of $72 \%$, specificity of $66 \%$ and negative predictive value of $82 \%$ ).

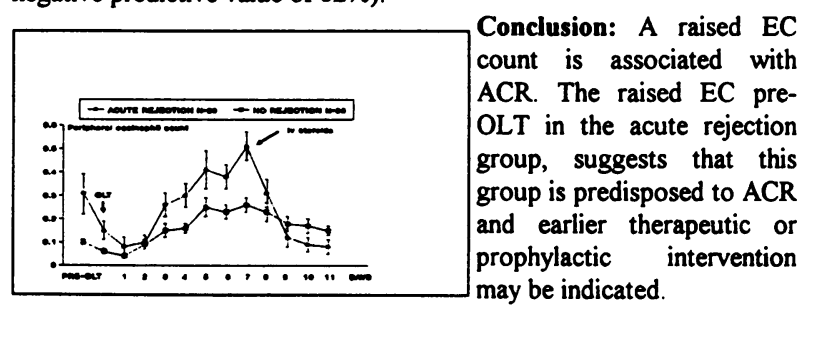

\section{Colorectal (clinical) W32-W43}

W32

\section{CHAOS THEORY, ARTIFICIAL NEURAL NETWORKS AND PROGNOSTICATION IN COLORECTAL CANCER. \\ P.J.Drew. ${ }^{ \pm}$L.Bottaci. R.Farouk.P.W.R.Lee ${ }^{\prime}$ L.M.C. Macintvre \\ G.S.Duthie LR.T.Monson. The University of Hull. Academic Surgical Unit, Castle Hill Hospital \& ${ }^{+}$Department of Computer Science, Hull,} U.K. \& "The Western General Hospital. Edinburgh. U.K.

The prediction of survival following surgery for colorectal cancer is unreliable and current techniques are only able to provide population survival figures. Artificial intelligence programmes have previously been unable to provide a consistently superior performance. Biological systems commonly represent "non-linear" Complex Adaptive Systems and the inability of traditional "linear" analysis to accurately assess outcomes in these systems is predicted by the emergent science of Chaos Theory. Artificial neural networks represent an alternative form of artificial intelligence which, by simulating a biological sy'stems" nonlinear, parallel computational techniques, are able to "learn" from established outcome data. This facilitates a level of flexibility and accuracy of pattern recognition in complex systems impossible to achieve with conventional analysis.

We investigated the ability of neural networks to prognosticate for individual patients following surgery for colorectal cancer.

Five year follow up data on 334 colorectal cancer patients was used to teach and validate the networks. Several network designs were constructed: 41 input parameters made up the first layer of neurones, 12 15 neurones the second with the output neurone providing a yes $/ n o$ answer. The trained networks were then tested using 2 year follow up data from 92 randomly selected patients from another hospital.

The networks achieved an overall accuracy of greater than $80 \%$ for the prediction of cause specific mortality from colorectal cancer at 12 months in the validation data set and $90 \%$ for cause specific mortality at 12 months for the patients from the alternative institution.

We conclude that developments in non-linear dynamics and artificial neural network technology' will facilitate accurate individual prognostication in colorectal cancer. 
COLORECTAL CANCERS OVEREXPRESS GELATINASES (MATRIX METALLOPROTEINASES-2 AND -9). S.L.Parsons, S.A.Watson, H.M.Collins, P.A.Clarke and R.J.C.Steele. Department of Surgery. University hospital, Nottingham. United Kingdom. NG7 2UH

The matrix metalloproteinases (MMPS) are overexpressed in certain types of cancers. Gelatinases (MMPs 2 and 9) have the tightest cortelation with tumour invasion and metastasis. There are several methods available to measure gelatinase, but gelatin zymography is the only technique which can differentiate between the active and inactive forms.

We performed zymography on 58 known concentrations of pure gelatinase and showed that, using computerised image analysis, the technique was quantitative within the $0-50$ ng range with a linear relationship $(r=0.813)$. Inter-gel variation was the main source of error but this was corrected for using known standards on all gels.

Gelatinase expression in $\mathbf{4 7}$ fresh frozen colorectal cancers and their corresponding normal mucosa was determined by Gelatin zymography. There was overexpression of the $92 \mathrm{kD}$ and $72 \mathrm{kD}$ Gelatinases and the active $62 \mathrm{kD}$ Gelatinase compared to normal mucosa $(\mathrm{p}<0.0001$, $p<0.0005, p<0.001$, paired Student's T-test). Gelatinase expression was higher in Dukes B compared to Dukes A ( $p<0.05$ for $72 \mathrm{kD}$ gelatinase), although this difference did not reach statistical significance for $92 \mathrm{kD}$ and $62 \mathrm{kD}$ gelatinase. There were no differences between gelatinase expression in Dukes B compared to Dukes C.

Gelatin Zymography is a useful and quantitative technique to detect gelatinase expression. Gelatinase expression is tightly correlated with the malignant phenotype in colorectal cancers.
The effect of pelvic radiotherapy on rectal compliance; a prospective study.

P.J. ius: J.H. Meerwaldt' (introduced by M. van Blankenstein). Deparment of Gastroenterology' and Radiotherapy ${ }^{2}$. Medisch Spectrum Twente, Enschede. the Netherlands.

Background: Following radiotherapy for pelvic tumours patients experience intestinal side effects. Changes in stool frequency combined with faecal urge or incontinence are the most common complaints.

We postulated the hypothesis that these complications are a result of a reduced rectal compliance (RC). The present study investigated this hypothesis prospectively.

Patients and methods: 30 patients with a carcinoma of the prostate were included. According to the extent of the disease two protocols were used:

$T_{i-2} N_{x-i} G_{1.2}$ received $59.8 \mathrm{~Gy}$ (13 patients).

$T_{1 .:} N_{x: 1} G_{3}$ and $T_{2<} N_{x-1} G_{1.3}$ received 64,4 Gy (17 patients).

The volume of rectum irradiated was expressed as recp95 (the percentage of the rectum within the $95 \%$ isodose-curve).

We used a highly compliant balloon and measured in the rectum the pressure in relation to the volume injected into the balloon. From these data a RC was calculated. Another parameter was the maximal tolerable volume (MTV).

Measurements were performed prior to radiotherapy and thereafter at sixmonthly intervals. At each measurement the patients were asked a series of questions regarding their stool habits.

Results: At 6, 12 and 18 months the mean RC decreased from 5.7 $\mathrm{ml} / \mathrm{cmH}_{2} \mathrm{O}$ to respectively $4.6,4.3$ and $4.6 \mathrm{ml} / \mathrm{cmH}_{2} \mathrm{O}$. The $\mathrm{MTV}$ decreased at the same time from $298 \mathrm{ml}$ to 245,245 and $228 \mathrm{ml}(p=0.0001: \mathrm{RC}$ and MTV before and after treatment). With increasing doses of radiotherapy there was a significant decrease in $R C(p=0.01)$.

The MTV changes were strongly related to the recp95 $(p=0.001)$

The mean stoolfrequency increased from 7.6 to 9.6. 10.9 and 11 times/week.

Conclusion: Increased stoolfrequency after pelvic radiotherapy is linked to reduced rectal compliance. There is a clear correlation between the dose of radiotherapy, the recp95 and the decreased RC.
Pilot Study for a Multicentre Randomised Trial of 'Onra. Only' Flexible Sigmoidoscopy (FS) for Prevention of Bowel Cancer Atkin WS*, McIntyre P**, Aubrey $\mathrm{R}^{* *}$, Cuzick J***, Edwards $\mathrm{R}^{* * *}$, Northover JMA*.

"Imperial Cancer Research Fund, St Marks Hospital Middx** Queen Elizabeth 11 Hospital, Welwyn Garden City, Herts. *** ICRF Dept Mathematics, Statistics, Epidemiology, Lincoln's Inn Fields, London.

We are planning a multicentre randomised controlled trial to evaluate the effect of a single FS at age 55-64 years in the prevention of colorectal cancer morbidity and death. It is intended to recruit 195,000 men and women to the study in 10 centres throughout the UK and randomly allocate to screening or control groups in the ratio of 1:2. To increase compliance rates, subjects will be eligible for entry into the study if they respond to a questionnaire and indicate that they would have the test if invited. The control group will not be contacted. The screened group will be invited for a single FS. Small polyps will be removed during screening. Those with 'high-risk' polyps $(\geq$ 3 adenomas, size $\geq 1 \mathrm{~cm}$, tubulovillous or villous histology or severe dysplasia) will undergo colonoscopy follow-up. Those with no or 'low risk' polyps will be discharged.

We undertook a pilot study of the protocol examining the recruitment procedure, acceptability of information supplied to participants, attendance rates, logistics, yield of neoplasia, referral rates for colonoscopy and complication rates. Included were 3540 patients, aged 55-64 years, of 2 general practices in Welwyn Garden City. 2696 (76\%) responded to the initial questionnaire and $2093(59 \%)$ stated they would have the test if invited. 719 subjects were randomly allocated to the screening arm and $533(74 \%)$ attended. Subjects' experience of pain was: none $30 \%$, mild $51 \%$, moderate $18 \%$, severe $2 \%$. Polyps were detected in $25 \%$ and adenomas in $9 \%$ of screenees. 30 people (5.6\%) had high risk adenomas and underwent colonoscopy. Four cancers were detected. The pilot study confirmed that the trial protocol is logistically feasible and acceptable. FOBTs) went on to FOS whereas 314 refused FOS at the GP surgery and 409 did not attend for the GP appointment.

Conclusion: Compliance figures with FOBTs in this population are of the same order as those previously published from the UK. However, the very poor compliance with FOS needs to be addressed and certainly allowed for in calculations for future screening studies. 
POTENTIAL COSTS AND BENEFITS OF ONCE-ONLY SIGMOIDOSCOPY FOR COLORECTAL CANCER SCREENING. HE Mulcahy, DP O'Donoghue, D Sethi, R Garlic, PD Fairclough, MJG Farthing. Digestive Diseases Research Centre, St Bartholomew's and Royal London School of Medicine and Dentistry, London, U.K., Gastroenterology \& Liver Unit, St Vincent's Hospital, Dublin, Ireland and East London \& City Health Authority, London, U.K.

Sigmoidoscopy with concomitant removal of adenomatous polype is known to reduce colorectal cancer incidence and mortality, and once-only $60 \mathrm{~cm}$ flexible sigmoidoscopy hes been proposed an an effective method of screening the a-risk population. However, few dect are available on the potential cost of this form of screening. Our aim was to estimate the cost of once-only sigmoidoscopic screening at 60 years in a defined population in the "East London and City" area, England.

Population data were obtained from the Office of Population Census and Surveys. Incidence and demographic data were obtained from the 'Thames Cancer Registry', covering the South-East area of Englend. Pathological, treatment and survival data were obtained from a prospective database containing over 1,200 consecutive colorectal cancer cases (SVH colorectal cancer database).

All figures and costs are 1995 estimates and assume $72 \%$ compliance with screening. In the 602,247 strong East London and City population, 3,360 sigmoidoscopies would be required annually to screen the 60 year old population. In addition, 135 total colonoscopies would be required to examine the proximal bowel of those patients with a significant index lesion in their distal bowel. Endoscopy would prevent or favourably influence staging of $18 \%$ (36/197) of all expected cancers and result in 21 lives being saved per year in the at-risk population. The total costs for endoscopy and initial endoscopic treatment are estimated at $£ 367,000(\$ 576,000)$ per year. The cost per colorectal cancer death avoided is estimated at $£ 17,500(\$ 27,500)$.

We conclude that the estimated cost-benefit ratio of once-only flexible sigmoidoscopic screening compares favourably with estimates for both breast and cervical cancer screening programmes.
PROSPECTIVE EVALUATION OF CONTRAST ENHANCED MAGNETIC RESONANCE IMAGING IN THE EVALUATION OF FISTULA IN ANO

IJ Beckingham , C Adams", GW Dyke; JA Spencer ${ }^{\dagger}, J^{\circ}$ Ward ${ }^{\dagger}$, NS Ambrose

Departments of Surgery and Radiology ${ }^{\dagger}$, St James Hospital Leeds.

The key to the successful management of fistula in ano lies in the identification of the relationship of the primary track to the anatomical structures through which it passes, and of additional tracks and abscesses which may prevent resolution of the primary track.

We prospectively compared a dynamic contrast enhanced magnetic resonance imaging (MRI) sequence with digital rectal examination. operative findings and long term follow up in the evaluation of primary fistula tracks, secondary tracks and abscesses in $\mathbf{4 2}$ consecutive patients with a suspected diagnosis of fistula in ano.

Contrast enhancement shows granulation tissue as a high signal intensity which clearly highlighted active tracks against the control 1 weighted images. There were five discordance's between MRI and surgery in the identification of primary tracks: MRI showed an ischio rectal abscess and track with no enteric connection in one patient who at operation had an epithelialised primary fistula. Four patients with fistulae on MRI had no enteric opening found at surgery and were treated as sinuses. Long term follow up has shown failure to heal in all four patients and further surgery has confirmed fistulae in the sites predicted by MRI.

When compared with final outcome measures (as opposed to surgical findings alone) MRI had a $97 \%$ sensitivity and $100 \%$ specificity in the detection of fistulae. MRI also identified more secondary tracks and was more accurate at identifying complex fistulae than either digital examination alone or surgical exploration.
Experience of the Jass prognostic system for cancer of the rectum within the Wessex Colorectal Cancer Audit JA Grabham, MG Coleman, D Bailey, CEH du Boulay, MR Thompson

In 1987, Jeremy Jass and colleagues published a new prognostic classification system for rectal cancer which they believe to be superior to the staging system of Cuthbert Dukes. This study applies the Jass scoring system to rectal tumours managed within the Wessex Colorectal Cancer Audit.

Between September 1991 and August 1993, 777 cases of rectal cancer without distant metastases underwent resection. Of these, 292 tumours had data recorded sufficient to allow asssignment of a Jass score. Early results (up to 2 years follow-up) for corrected survival and local recurrence suggest that the Jass system is a valid prognostic method with patterns following those of the Dukes' scores. The principle difference between the two systems supports the claimed advantage of the Jass system to consign more patients into groups that allow a confident prediction of clinical outcome ( $41.4 \%$ of tumours were in Jass groups 1 or 4 whereas $24.3 \%$ were Dukes' A or C2).

However, concerns have been raised regarding the objectivity of two of the central components of the Jass system - the classification of the invading margin into infiltrating or expanding, and the presence or absence of a lymphocytic infiltrate. In this series, $67.8 \%$ of tumours were judged to have an infiltrating mangin (compared with $20-30 \%$ as suggested by Jass) and $37.3 \%$ of tumours had a lymphocytic infiltrate (nearly twice as frequent as in the Jass series).

These results appear to reinforce doubts regarding the reproducibility of the Jass classification system and suggest that its prognostic value may rely on those variables which it shares with the Dukes' system.
CONTINENCE FOLLOWING LOW ANTERIOR RESECTION NEORECTAL DYNAMICS AND ANAL PHYSIOLOGY. G.N.Rao.P L.Drew, A. Gardiner. R.Farouk. P.W.R.Lee, J.R.T.Monson, G. S. Duthie. The

University of Hull Academic Surgical unit, Castle Hill Hospital, Hull, U.K.

A previously validated model for the assessment of rectal physiology based on accurate in vivo ultrasonic measurements and established physical principles was utilised for the analysis of neorectal dynamics and continence following low anterior resection.

Twenty four patients who had undergone low anterior resection were recruited. Three groups were identified according to their functional score: Major incontinence (score 4-7) $(n=5)$, Minor incontinence (score $2-3)(n=8)$, and Normal continence $(n=11)$. These groups were comparable in terms of clinical and anastomotic characteristics and anal squeeze and resting pressure.

With an anastomosis at or below $7 \mathrm{~cm}$ from the anal verge rapid inflation of the rectal balloon caused a sustained drop in anal pressure with no evidence of recovery. This prolonged anorectal inhibition was not seen with anastomoses above $7 \mathrm{~cm}$. Major incontinence was associated with a high neorectal wall stiffness and disordered viscoelastic parameters with evidence of decreased elasticity within the neorectum. Strain work was increased at all rectal sensations. Sequential multivariate analysis of the models parameters revealed the smooth muscle component to be significantly $(p=0.01)$ increased. This study demonstrates that major incontinence following low anterior resection is due to a prolonged rectoanal inhibitory reflex associated with increased neorectal wall stiffness caused by increased neorectal smooth muscle activity. 
RECTAL TRANSIT IN PATIENTS WITH DISORDERED DEFAECATION. Kumar D Benson $\mathrm{M}^{*}$, Grant E, Britton $\mathrm{A}^{\prime \prime}$, Gains $G^{\prime}$, Jazrawi $R^{*}$, Joseph $A^{\prime}$. Departments of Colorectal Surgery, Gastroenterology* and Radiology", St George's Hospital, Tooting, London, UK.

Assessment of rectal transit is commonly performed using barium proctography. The result obtained from such measurements only reflect total percentage evacuation from the rectum and do not take into account the rate of evacuation. The aim of this study was to evaluate the impact of the rate of evacuation and total.

We studied 104 patients with a history of constipation. Rectal transit was measured using isotope defaecography in an unprepared rectum. Gamma camera images were obtained at 5 second intervals during the act of defaecation. The rectal transit was normal in 29 patients with a total percent evacuation of $>70 \%$ and a rate of evacuation of $>1.5 \%$ per second [70.5 (3.5), $1.5(0.22)]$. There were no associated anatomical abnormalities such as a rectocele in this group. The remaining 75 patients had impaired rectal transit. The total percent evacuation in this group was significantly lower than the one with $>70 \%$ evacuation $(70.5(3.15)$ vs $60.5(1.9)$. The rate of rectal evacuation in those with reduced percent evacuation was significantly slower $(p<0.05)$ than those with normal evacuation. There was a significant correlation between the rate of evacuation and total percentage evacuation $(r=0.46$, $p<0.0001$ ). There was an inverse correlation between the rate of evacuation and the presence of a rectocele $(r=0.28, p<0.005)$.

These data suggest that the rate of evacuation is an important factor in the dynamics of defaecation and should be routinely measured in patients with disordered defaecation.
COIL EMBOLISATION - A SAFE AND EFFECTIVE OPTION FOR MAJOR COLONIC BLEEDING.

A.A.Nicholson*,J,E.Hartley,I.Curzon*,D.F.Ettles*,G.S.Duthie

P.W.R.Lee,J.R.T.Monson. The University of Hull, Academic Surgical Unit Castle Hill Hospital, Cottingham HU16 5JQ, and *Dept. of Radiology, Hull Royal Infirmary, Anlaby Road, Hull HU3 $2 \mathrm{JZ}$.

The management of major colonic bleeding is problematical. Although the majority of patients settle with conservative measures, a proportion of patients require emergency surgery which is associated with high morbidity and mortality. Selective angiography can localise the source of haemorrhage in actively bleeding patients prior to surgery. However, therapeutic angiography, though technically feasible, has not gained widespread support.

Since January 1995 eight patients (2 males, 6 females mean age 81yrs, range 72-87yrs) with major colonic bleeding have undergone coil embolisation of bleeding points. All patients had failed to respond to conservative measures (mean haemoglobin at angiography $6.5 \mathrm{~g}$ despite mean transfusion volume of 6 units), were haemodynamically unstable at the time of angiography (mean systolic pressure $85 \mathrm{mmHg}$ ), and were destined for emergent laparotomy should embolisation fail. The source of bleeding was identified in all patients. In 5 patients single bleeding "end-vessels" were embolised with a single coil, with arrest of haemorrhage. None of these patients required further intervention for bleeding. A 6th patient with Crohn's disease required surgery for continued bleeding, despite embolisation of multiple left colonic bleeding points. A further patient had 2 discrete bleeding points successfully embolised at the caecum and hepatic flexure. Subsequent barium enema and colonoscopy with biopsy revealed an ischaemic stricture at the hepatic flexure, from which the patient is asymptomatic. The final patient developed ischaemic colitis after embolisation had arrested haemorrhage from the left colon. On this occasion, in contrast to the earlier cases, the bleeding "end-vessel" could not be entered, and the marginal artery was therefore embolised. This latter complication was managed conservatively.

This preliminary experience demonstrates a role for coil embolisation in the management of patients with major colonic haemorrhage. Significant colonic ischaemia is unlikely provided the marginal artery is preserved. Given appropriate angiographic facilities and levels of expertise, the use of these techniques in selected groups of patients can obviate the requirement for emergency surgery in this notoriously high risk scenario.

\section{Helicobacter pylori W44-W53}

W44

FAECAL INCONTINENCE IN PATIENTS WITH NORMAL ANAL SPHINCTERS - EVIDENCE FOR AN AFFERENT DEFECT. Benson $M^{*}$, Grant $E$, Jazrawi $R^{*}$, Lloyd $R^{*}$ Leicester RJ. Kumar D. Departments of Gastroenterology* and Coloproctology, St George's Hospital, Tooting, London, UK.

Patients with faeca! incontinence (FI) often have impaired function of either the internal (IAS) and/or external (EAS) anal sphincters. The underlying cause is either a primary neuropathy or traumatic disruption of the anatomical integrity of the sphincter(s). However, a proportion of patients have no demonstrable physiological dysfunction of either the IAS or EAS. The pathophysiological mechanism of in this subgroup remains unclear.

Method: In the last 15 months, 134 patients with FI (median age 57 [IQR:34-68]) underwent anorectal physiological measurement. Sphinteric basal (BP) and maximum squeeze (SP) pressures were measured using water-filleu microballoon system. The average of 4 separate measurements was calculated for both variables. Rectal sensation was assessed by filling an intra-rectal latex balloon with $50 \mathrm{ml}$ aliquots of air at 60 s intervals. Values were established for the threshold volume (TV), volume of constant sensation (VCS) and maximum tolerated (MTV). Endoanal ultrasound was also performed on all patients.

Results: The basal and squeeze pressures were normal in 27 of $134(20 \%)$ patients with FI. Endoanal ultrasound was also normal and did not reveal sphincter defect in all these 27 patients. The rectal threshold sensation was normal in all patients. Seven of 27 patients $(25 \%)$ showed an abnormal constant volume sensation $(<120-150 \mathrm{mls})$. The remaining $20(75 \%)$ had impairment of VCS as well as MTV $(<200-220 \mathrm{mls})$. None of the patients had impairment of MTV alone.

These data suggest that in patients with a manometrically and morphologically normal anal sphincters, abnormalities of rectal sensation may be responsible for faecal incontinence.
SYMPTOMS, CONSULTATION, MEDICATION AND WORKLOSS AFTER ERADICATION OF H. PYLORI: A THREE YEAR FOLLOW-UP OF DUODENAL ULCER T.G.Reilly, D Stone, RP Walt. University Department of Medicine, Queen Elizabeth Hospital Birmingham.

Introduction: We have previously shown benefit following eradication of Helicobacter pylori in ulcer patients. We have extended the follow-up in this cohort of ulcer patients.

Methods: A validated questionnaire was sent to patients treated for ulcer by eradication therapy and tested at 4 weeks by ${ }^{13} \mathrm{C}$-urea breath test, asking details of symptoms, consultations with doctors, use of medication including antisecretory drugs and NSAIDs, and breath test.

Results: From 203 patients sent a repeat questionnaire 22-52 months (mean $31 \pm 6.0)$ after treatment, 140 (69\%, 109 Hp negative) replies were received. The mean age of responders was 56 yrs $( \pm 13.8$, range 27-83). There was no significant difference between $\mathrm{Hp}$-pos and Hp-neg in terms of age, sex, length of follow-up, use of tobacco and NSAIDs, or response rate. The results of the questionnaire were:

Current 'ulcer pain'

Belief that ulcer was cured

Treatment in last year for ulcer

Consulted GP in last year about ulcer symptoms

Lost work (if in work last 18/12) due to ulcer

\begin{tabular}{ccc} 
Eradicated & Failed & $\chi^{2}$ test \\
\hline $15 / 107$ & $12 / 29$ & $\mathrm{p}=0.0021$ \\
$92 / 106$ & $10 / 30$ & $\mathrm{p}<0.0001$ \\
$12 / 103$ & $18 / 30$ & $\mathrm{p}<0.0001$ \\
$10 / 109$ & $16 / 31$ & $\mathrm{p}<0.0001$ \\
$3 / 51$ & $7 / 15$ & $\mathrm{p}=0.0005$ \\
& & (Yates)
\end{tabular}

Eradication successes had the same frequency of heartbum and irritable bowel symptoms as failures. Heartburn was associated with 'ulcer pain' $(p=0.039)$, current use of medication $(p<0.0001)$ and belief that the ulcer was not cured $(\mathrm{p}=0.017)$. Those with heartbum were not more likely to report current NSAID usage than controls $(p=0.82)$. Of 51 (9 positive) patients who had a repeat controls $(p=0.82)$. Of 51 ( 9 positive) patients who had a repeat
breath test, one previously negative patient became positive.

Conclusions: Eradication of $H$. pylori in patients with peptic ulcer gives long term benefits. Heartburn is more often a feature in those with continued symptoms than those without, regardless of H.pylori status. A 4-week breath test is a good predictor of long term H.pylori status. time lost from work. All responders were invited to repeat the 